\title{
A core signaling mechanism at the origin of animal nociception
}

\author{
Oscar M. Arenas ${ }^{1}$, Emanuela E. Zaharieva ${ }^{1}$, Alessia Para ${ }^{1}$, Constanza Vásquez-Doorman ${ }^{2}$,
} Christian P. Petersen² and Marco Gallio ${ }^{1 *}$.

\author{
Affiliations: \\ ${ }^{1}$ Department of Neurobiology, \\ ${ }^{2}$ Department of Molecular Biosciences, Northwestern University, Evanston, Illinois, USA. \\ *Correspondence to: marco.gallio@northwestern.edu
}

All animals must detect noxious stimuli to initiate protective behavior, but the evolutionary origin of nociceptive systems is not well understood. Here, we show that a remarkably conserved signaling mechanism mediates the detection of noxious stimuli in animals as diverse as flatworms and humans. Planarian flatworms are amongst the simplest bilateral animals with a centralized nervous system, and capable of directed behavior. We demonstrate that noxious heat and irritant chemicals elicit robust escape behaviors in the planarian Schmidtea mediterranea, and that the conserved ion channel TRPA1 is required for these responses. TRPA1 mutant fruit flies (Drosophila) are also defective in the avoidance of noxious heat ${ }^{1-3}$. Unexpectedly, we find that either the planarian or the human TRPA1 can restore noxious heat avoidance to TRPA1 mutant Drosophila, even though neither is directly activated by heat. Instead, our data suggest that TRPA1 activation is mediated by $\mathrm{H}_{2} \mathrm{O}_{2} /$ Reactive Oxygen Species, early markers of tissue damage rapidly produced as a result of heat exposure. Together, our data reveal a core function for TRPA1 in noxious heat transduction, demonstrate its conservation from planarians to humans, and imply that human nociceptive systems may share a common ancestry with those of most extant animals, tracing back their origin to a progenitor that lived more than $\mathbf{5 0 0}$ million years ago. 
1 Each animal group uses specialized sensory systems to detect and avoid predators, find food

2 sources and mates. Due to specific demands, sensory systems evolve independently in different

3 species. Yet, while each animal group lives in a sensory world that is essentially unique, most of what

4 we know about sensory representation comes from a very limited number of species - a handful of

5 vertebrate and invertebrate model systems.

The detection of potentially harmful conditions is a core sensory task. The ion channel TRPA1 is remarkably conserved across animal evolution and has been implicated in the response to a broad range of electrophilic irritant chemicals as well as to noxious hot or cold temperature in humans ${ }^{4}$, mice $^{5-11}$, and flies ${ }^{2,3,12}$. Interestingly, while TRPA1's sensitivity to irritant chemicals has been widely conserved $\left({ }^{13}\right.$, in all but the $C$. elegans homolog $\left.{ }^{14}\right)$, its temperature gating appears to have changed repeatedly during evolution ${ }^{15}$. In vitro, some mammalian TRPA1 homologs are activated by noxious cold $^{16}$, while others are insensitive to temperature (reviewed in ${ }^{15}$ ). In contrast, TRPA1 from chicken ${ }^{17}$, various reptiles, and Xenopus frogs are activated by warm temperatures ${ }^{18,19}$, and the zebrafish genome encodes two distinct paralogs, only one of which shows thermosensory responses ${ }^{20,21}$. The situation in invertebrates is also complex: while $C$. elegans TRPA1 is activated by cold ${ }^{14}$, insect TRPA1s (honeybee ${ }^{22}$, mosquito ${ }^{23}$ etc.) are activated by warm temperatures, and the fruit fly homolog is spliced into at least four variants ${ }^{3}$, including both heat-sensitive and heat-insensitive ones $3,12,24$

What is the ancestral function of TRPA1? How ancient is its association with nociceptors? Planarian flatworms are an attractive system in which to study evolutionary origins of sensory transduction. As members of the phylum Platyhelminths, planarians are considered among the simplest animals with bilateral symmetry and a centralized nervous system. From an evolutionary perspective, they are nearly equally distant from taxa that include species extensively studied such as nematodes, flies and mice $\left({ }^{25}\right.$ and Figure 1a). Furthermore, recent work on regeneration has led to the development of RNAi protocols to systemically knock-down the expression of selected genes in 
bioRxiv preprint doi: https://doi.org/10.1101/185405; this version posted September 12, 2017. The copyright holder for this preprint (which was not certified by peer review) is the author/funder, who has granted bioRxiv a license to display the preprint in perpetuity. It is made available under aCC-BY-NC-ND 4.0 International license.

vivo $^{26}$. Here, we use these tools to investigate the function of TRPA 1 in the freshwater planarian

Schmidtea mediterranea.

A fragment of the S. mediterranea TRPA1 gene has been previously used in in situ hybridization experiments as a marker for a subset of differentiated neurons ${ }^{27}$. Starting from it, we cloned a full-length coding sequence for the gene (see methods for details), henceforth referred to as Smed-TRPA1 (Figure 1b). To test whether Smed-TRPA1 mediates the avoidance of noxious heat in S. mediterranea, we designed a two-choice avoidance assay (Extended Data Figure 1) based on the one we previously developed for fruit flies ${ }^{28}$. Animals were introduced into a small circular chamber covered by a thin film of water, and tracked while making a choice between floor tiles kept at moderate $\left(24^{\circ} \mathrm{C}\right)$ or hot $\left(32^{\circ} \mathrm{C}\right)$ temperatures; the time spent in each quadrant was then quantified to calculate an avoidance index (AI). In this assay, S. mediterranea showed robust avoidance of heat $\left(32^{\circ} \mathrm{C}, \mathrm{Al} 1\right)$, manifested as sharp turns away from the hot quadrants (Figure 1c). This is consistent with a nocifensive behavior, and indeed with the fact that $S$. mediterranea comes from cool water environments and can die from brief exposure to $35^{\circ} \mathrm{C}\left({ }^{29,30}\right.$ and data not shown).

Remarkably, the avoidance of hot quadrants was severely disrupted by RNAi knock-down of Smed-TRPA1 (Figure 1c-d and see Extended Data Video 1). Smed-TRPA1 RNAi animals glided around the chamber without turning at the hot-cold boundaries (see tracks in Figure 1c), and ended up spending nearly equal time in hot as in cool quadrants. This is in sharp contrast with the behavior of both untreated and control worms (i.e. worms fed dsRNA targeted to a sequence not present in the worm genome, Figure 1c-d). Importantly, Smed-TRPA1 RNAi worms glided around the chamber at comparable speed as controls (Figure 1e), and displayed robust negative phototaxis when given a choice between light and dark (in an independent assay, see Extended Data Figure 2), indicating that Smed-TRPA1 RNAi does not mar gross locomotor functions, nor does it impact all aversive behavior. 
RNAi knock-down of the transcription factor AP2 has been previously shown to impair the

expression of TRPA1 in S. mediterranea ${ }^{27}$. Based on this, we reasoned that ap2 RNAi could provide

an independent means to assess the role of TRPA1 in heat nociception. Unexpectedly, ap2 RNAi

animals did not display an avoidance defect, and instead avoided the hot quadrants as robustly as controls (Figure 1c-d). In situ hybridization revealed that ap2 RNAi was effective in knocking-down Smed-TRPA1 expression only within the brain, and not in peripheral neurons (Figure $\mathbf{1} \mathbf{f - k}$ ). The fact that animals with significantly reduced Smed-TRPA1 expression within the brain behave normally suggests that Smed-TRPA1 is required at the periphery for the detection or responses to noxious heat.

Next, we tested Smed-TRPA1 knock-down animals for potential defects in chemical nociception, by assaying behavioral responses to Allyl isothiocyanate (AITC). AITC is the agent responsible for the pungent taste of mustard and wasabi, and a well-known chemical agonist of TRPA ${ }^{6,7,11}$. We developed an arena consisting of circular chambers interconnected by small corridors that are not readily traversed by the worms (Figure 2a). Animals fed control dsRNA (see above) or Smed-TRPA1 dsRNA were introduced in the first chamber in the presence of a mock agar pellet, or -alternatively- of an agar pellet laced with AITC; their behavior was then monitored for 5 minutes. Mock pellets were readily explored by untreated animals as well as by RNAi controls, which as a result remained in their vicinity. In contrast, AITC produced strong aversive responses including rapid withdrawal and abrupt turns. The worms ultimately escaped away from the chamber containing the pellet traversing the narrow corridors (Figure 2b and Extended Data Video 2). Again in sharp contrast to controls, Smed-TRPA1 knock-down animals did not display aversive responses and instead remained in the first chamber, in the vicinity of the AITC-laced pellet (Figure 2b).

Our experiments show that Smed-TRPA1 is a key mediator of both heat avoidance and chemical nociception in vivo in S. mediterranea. To begin studying the biophysical properties of the channel in vitro, we next performed whole cell patch-clamp electrophysiology on heterologously 
expressing cells. To achieve functional expression of Smed-TRPA1, we chose Drosophila S2 cells, a system previously used for Drosophila TRPA ${ }^{31}$. Our recordings show that, in S2 cells, Smed-TRPA1 was activated by AITC (Figure 3). In contrast, the channel was not directly gated by heat (Figure 3ad). Even when mis-expressed in vivo, in transgenic Drosophila (i.e. in all fly neurons), Smed-TRPA1 could be readily activated by AITC, but not heat (Figure 4). As a control, the Drosophila TRPA1-A variant ${ }^{3}$ was activated by both AITC and heat in both contexts (Figure 3c-d and Figure 4, and ${ }^{32}$ ). The lack of thermal sensitivity of Smed-TRPA1 in vitro (vis-à-vis the effect of RNAi on noxious heat avoidance) appears puzzling. However, TRPA1 is well known to function both as a primary temperature receptor as well as a signal transduction component, i.e. downstream of diverse signaling events ${ }^{10,33}$. Interestingly, both the heat-nociception phenotype of TRPA1 mutant fly larvae and heat-entrainment defects of adults were readily rescued by a non-heat-sensitive variant of the fly TRPA1 (TRPA1-C, ${ }^{3,34}$ ). These observations led us to test directly the possibility that the non-heatsensitive Smed-TRPA1 may also be able to substitute for the fly TRPA1 -i.e. to attempt acrossphylum rescue of a Drosophila TRPA1 mutant by expression of the planarian homolog.

First, we used a rapid 2-choice assay for temperature preference ${ }^{28}$ (similar to that described above), and probed the responses of wild type and TRPA1 Drosophila mutants to both innocuous $\left(30^{\circ} \mathrm{C}\right)$ and noxious heat $\left(40^{\circ} \mathrm{C}\right.$, Figure $\left.5 \mathrm{a}\right)$. Consistent with previous reports ${ }^{2}$, in our assay TRPA 1 mutant flies showed a clear defect in the avoidance of noxious heat (Figure 5a,c; note that residual heat avoidance is likely mediated by GR28b.d -a distinct molecular hot receptor ${ }^{35}$ ). Much like in the larva, this nociceptive phenotype could be significantly rescued by ubiquitous expression of TRPA1C, a TRPA1 variant not directly activated by heat ${ }^{3}$ (Figure 5 b,c). Strikingly, a comparable amount of rescue could be achieved by ubiquitous expression of the planarian Smed-TRPA1 (34\% identical and $53 \%$ similar to the fly TRPA1 in amino-acidic sequence; Figure $5 \mathbf{b}, \mathbf{c}$ ), and even of a human TRPA1 cDNA ( $36 \%$ and $31 \%$ identical, and $57 \%$ and $49 \%$ similar to fly and Smed-TRPA1, respectively; Figure $\mathbf{5 b , c )}$. The rescue of the fly phenotype by an evolutionary distant 'heat-insensitive' homolog 
such as Smed-TRPA1, and the human TRPA1 (activated by cold rather than heat), argues that the function of TRPA1 in heat nociception is unlikely to be fully explained by direct heat gating.

Instead, a number of observations point towards early markers of tissue damage as potential mediators of TRPA1 activation during nociceptive heat responses. Hydrogen peroxide $\left(\mathrm{H}_{2} \mathrm{O}_{2}\right)$, is amongst the earliest known markers of mechanical tissue damage in vertebrates ${ }^{36}$, Drosophila ${ }^{37}$ as well as Planarians ${ }^{38} . \mathrm{H}_{2} \mathrm{O}_{2}$ is a well-known activator of mammalian and Drosophila TRPA1 $\left({ }^{39-43}\right.$, together with additional Reactive Oxygen Species $-\operatorname{ROS}^{44}$ ), and recent work suggest that responses to potentially damaging short-wavelength UV light occurs through photochemical production of $\mathrm{H}_{2} \mathrm{O}_{2}$, and requires TRPA1 in both flies ${ }^{41-43,45}$ and Planarians ${ }^{46}$. Thus, if noxious heat were to cause rapid, localized, production of $\mathrm{H}_{2} \mathrm{O}_{2} / \mathrm{ROS}$, this could provide the direct signal for TRPA1 activation that mediates nociceptive responses.

For this hypothesis to be correct, a number of conditions have to be met: (1) SmedTRPA1 (like human and Drosophila) should be activated by $\mathrm{H}_{2} \mathrm{O}_{2} ;$ (2) In vivo, heat stimulation in the appropriate range should cause rapid $\mathrm{H}_{2} \mathrm{O}_{2} / \mathrm{ROS}$ production (on a timescale compatible with the animal's escape behaviors) and, (3) if indeed nociceptive heat responses are mediated by $\mathrm{H}_{2} \mathrm{O}_{2} / \mathrm{ROS}$, an acute increase in $\mathrm{H}_{2} \mathrm{O}_{2} / R O S$ levels should sensitize the animal's behavioral responses to noxious heat, and this sensitization should depend on TRPA1. Our experiments confirm each of these predictions.

First, we tested Smed-TRPA1 for potential responses to $\mathrm{H}_{2} \mathrm{O}_{2}$ in vitro in our cell expression system (see above). Our recordings showed that, in S2 cells, Smed-TRPA1 was indeed activated by a range of $\mathrm{H}_{2} \mathrm{O}_{2}$ concentrations (Figure 6a,b), as was the Drosophila counterpart TRPA1-C (Figure 6b; i.e. the 'heat insensitive' fly variant which also supported behavioral rescue in our experiment). We note that, while it is difficult to speculate on the $\mathrm{H}_{2} \mathrm{O}_{2} / \mathrm{ROS}$ concentration that TRPA1 may encounter during a heat challenge in vivo, secondary modifications (such as prolyl hydroxylation ${ }^{47}$ ) have been shown to dramatically increase TRPA1 responses to $\mathrm{H}_{2} \mathrm{O}_{2} / \mathrm{ROS}$, potentially expanding the effective sensitivity of the channel. 

under aCC-BY-NC-ND 4.0 International license.

Next, we tested if heat stimulation in the appropriate range (i.e. in the 'noxious' range for each species) may lead to the rapid production of $\mathrm{H}_{2} \mathrm{O}_{2} / \mathrm{ROS}$ in both Schmidtea and Drosophila tissue. For this experiment, we loaded living samples with the dye 5-(and 6)-carboxy-2',7'dichlorodihydrofluorescein diacetate (carboxy- $\mathrm{H}_{2}$ DCFDA, a widely used fluorogenic ROS marker for live cells ${ }^{48}$ ), and monitored potential fluorescence changes in response to heat exposure by confocal microscopy. As previously reported, live planarian worms could be directly loaded with the dye, and displayed $\mathrm{H}_{2} \mathrm{O}_{2} / \mathrm{ROS}$ induced fluorescence at sites of physical wounding $\left({ }^{38}\right.$ and data not shown; and see ED Figure 3 for additional controls). Fast $\mathrm{H}_{2} \mathrm{O}_{2} /$ ROS production was also detected when the worms were submitted to rapid heating while under the microscope (i.e. by using a heating stage; $\Delta t=20>35^{\circ} \mathrm{C}$, speed $=\sim 1^{\circ} \mathrm{C} / 4$ seconds). Consistent with the 'noxious' range for this animal $\left(30-35^{\circ} \mathrm{C}\right)$, we observed rapid $\mathrm{H}_{2} \mathrm{O}_{2} /$ ROS increases starting above $23-25^{\circ} \mathrm{C}$, and culminating in widespread fluorescence around $30-35^{\circ} \mathrm{C}$ (Figure 6c-e). Drosophila tissues also displayed rapid $\mathrm{H}_{2} \mathrm{O}_{2} / \mathrm{ROS}$ increases in response to heating, but this time the increase in fluorescence started around $30^{\circ} \mathrm{C}$, and culminated around $40-45^{\circ} \mathrm{C}$ (Figure 6f-g), consistent with the 'noxious' range for Drosophila (35$40^{\circ} \mathrm{C}$ ). Importantly, in both planarians and fly tissues, we recorded fluorescence changes rapid enough to be compatible with the timescale that would be required to trigger/modulate behavioral responses: for example between two imaging frames (Figure $6 \mathbf{d}$, i.e. separated by $\sim 300 \mathrm{~ms}$ ), or after as little as one second of exposure to heat (Figure 6h).

Carboxy- $\mathrm{H}_{2}$ DCFDA is a general oxidative stress indicator, and does not discriminate amongst different reactive oxygen species. To directly test if $\mathrm{H}_{2} \mathrm{O}_{2}$ in particular may be amongst the species produced during a noxious heat challenge, we turned to the genetically encoded $\mathrm{H}_{2} \mathrm{O}_{2}$ indicator roGFP2-Orp1. This indicator couples the redox-sensitive green fluorescent protein 2 (roGFP2) with the yeast $\mathrm{H}_{2} \mathrm{O}_{2}$ sensor Orp1 ${ }^{49}$, allowing the measurement of changes in $\mathrm{H}_{2} \mathrm{O}_{2}$ levels in intact living animals ${ }^{50}$. Our results show that, in transgenic Drosophila larvae, roGFP2-Orp1 reported a significant 
increase in $\mathrm{H}_{2} \mathrm{O}_{2}$ upon brief ( $\sim 5$ seconds) exposure to noxious heat (Figure ED4, see legend and methods for details).

Finally, we tested the notion that -if noxious temperatures are indeed sensed at least in part through $\mathrm{H}_{2} \mathrm{O}_{2} /$ ROS production- an acute, systemic increase in $\mathrm{H}_{2} \mathrm{O}_{2} / \mathrm{ROS}$ levels may sensitize the animal's behavioral responses to heat. Here, we tested adult Drosophila for heat avoidance using our 2-choice assay (see above), but this time the flies' performance in the arena was preceded by a short feeding with $\mathrm{H}_{2} \mathrm{O}_{2}{ }^{51}$ or Paraquat (a potent pro-oxidant ${ }^{52}$ ). Strikingly, pro-oxidant feeding significantly

TRPA1 mutant flies (Figure 6i). This result directly demonstrates that $\mathrm{H}_{2} \mathrm{O}_{2} /$ ROS can sensitize aversive responses to heat in Drosophila, and that this sensitization requires functional TRPA1 channels.

Planarian flatworms are a powerful, and yet underutilized model for behavioral research. They are capable of active hunting behavior, and possess simple sensory systems ${ }^{30,53}$ and a simple brain that operates using synaptic and neurotransmitter principles similar to those of the more complex insect or mammalian brains ${ }^{54}$.

Here, we have shown that the ion channel TRPA1 functions as a key transduction component for nociceptive signals in Schmidtea mediterranea. In insects and many vertebrates (snakes, frogs etc.), TRPA1 channels can be directly gated by temperature changes, but our work in Schmidtea and Drosophila suggests that TRPA1's function in nociceptive heat sensing goes beyond that of a canonical heat-activated ion channel. Instead, our data suggest that $\mathrm{H}_{2} \mathrm{O}_{2} / \mathrm{ROS}$ are rapidly produced in response to noxious heat, and that this signal contributes to channel activation to mediate defensive responses. We note that this mechanism provides an especially satisfactory explanation for the remarkable across-phylum rescue of heat-avoidance phenotypes of TRPA1 mutant Drosophila by both Schmidtea TRPA1 (insensitive to heat), as well as human TRPA1 (activated by cold). The heat range that is expected to produce tissue damage in Drosophila (i.e. the "noxious range", 35- 
$\left.40^{\circ} \mathrm{C}\right)$ is different from that of Schmidtea $\left(\sim 30^{\circ} \mathrm{C}\right)$ from that of human $\left(\sim 45^{\circ} \mathrm{C}\right)$-and yet transgenic

rescue of fly mutants restored heat avoidance to that of the host (Drosophila) rather than the channel

donor (Schmidtea or human). This can be easily accounted for by the fact that the thermal range that

causes heat damage (and therefore $\mathrm{H}_{2} \mathrm{O}_{2}$ /ROS production) is determined by the heat tolerance of the

178 host tissue.

Finally, our results suggest that early Bilaterians already possessed a polymodal

(chemical/thermal) nociceptive system that relied on $\mathrm{H}_{2} \mathrm{O}_{2} /$ ROS-mediated TRPA1 activation. This

core function has been conserved in extant lineages, and may have placed TRPA1 in a key position

to undergo the additional transitions into a hot- or cold-activated channel that have been documented

in different animal groups. Our results also imply that human pain systems may share a common

ancestry with the nociceptive systems of extant bilateral animals, tracing back their origin to the

common 'Urbilaterian' progenitor that lived more than 500 million years ago. 


\section{Methods}

\section{$\underline{\text { Cloning of a Smed-TRPA1 full length coding sequence }}$}

A full-length Smed-TRPA1 coding sequence was amplified by PCR starting from a Schmidtea mediterranea cDNA library. The library was generated by Superscript III reverse transcription (Life Technologies) from total RNA, purified from whole animals using Trizol followed by DNAse treatment with DNA-free (Ambion/ThermoFisher). The following primers were used for PCR: FWD 5'-

\section{CAaaacATGAATAAAATTTCTAAAAACCGAAAAACCTC-3' and REV 5'-}

TTAAAAATTGTTATCTGGTTTGACAGATTTCTG -3' (Kozak sequence for Drosophila in lower case letters). Analysis of 5'- and 3'-RACE libraries produced with SMARTer® RACE 5'/3' Kit (Clontech) confirmed that the identified sequence includes the appropriate ATG and stop codons and represents a single, full-length Smed-TRPA1 coding sequence (3510 bp). Analysis of available RNAseq data (SmedGD, http://smedgd.neuro.utah.edu/; see Robb et al., Genesis. 2015 Aug;53(8):535-46) indicates that Smed-TRPA1 likely produces a single transcript, encoding a protein of 1169 amino acids, which contains $14 \mathrm{~N}$-terminal ankyrin repeats (Interpro database entry: IPR002110) followed by an ion transporter domain (Interpro database entry: IPR005821).

\section{$\underline{\text { Phylogenetic analysis of TRPA1 homologs }}$}

A neighbor-joining phylogenetic tree was constructed using the full sequence of bona fide (experimentally validated) TRPA1 proteins from 25 organisms: A. gambiae (ACC86138), A. aegypti (AAEL009419), D. melanogaster (AEU17952), B. mori (NP_001296525), C. elegans (ABQ15208), C. brevicauda (AEL30802), C. porcellus (NP_001185699), C. hortulanus (ADD82932), C. atrox (ADD82930), D. rerio (NP_001007066 and NP_001007067), D. rotundus (AEL30803), G. gallus (NP_001305389), H. armigera (AHV83756), H. sapiens (NP_015628), M. mulatta (XP_001083172), M. musculus (NP_808449), P. obsoletus lindheimeri (ADD82929), P. jerdonii (AEW26660), P. regius 
(ADD82928), R. norvegicus (NP_997491), T. rubripes (XP_003968031), T. castaneum (LOC658860),

V. destructor (BAO73033 and BAO73034), X. tropicalis (BAM42680).

\section{Planarian RNAi}

The CIW4 asexual laboratory strain of Schmidtea mediterranea was used for all experiments.

Animals were kept in plastic containers filled with $1 \mathrm{x}$ artificial planarian water (APW) that contained:

$1.6 \mathrm{mM} \mathrm{NaCl}, 1 \mathrm{mM} \mathrm{CaCl}_{2}, 1 \mathrm{mM} \mathrm{MgSO}_{4}, 0.1 \mathrm{mM} \mathrm{MgCl}_{2}, 0.1 \mathrm{mM} \mathrm{KCl}, 1.2 \mathrm{mM} \mathrm{NaHCO}_{3}$. Planarians

were fed with homogenized calf liver for stock maintenance. The containers were cleaned two days after feeding or once a week if starved. Templates for RNA synthesis were generated by PCR from pGEM-t vectors (Promega) harboring $1.5 \mathrm{~kb}$ fragments of Smed-TRPA1 or Smed-AP2 cDNA; a $0.8 \mathrm{~kb}$ UNC22 PCR product was used to generate UNC22 template as an RNAi control (UNC22 is a C. elegans gene not present in the S. mediterranea genome). The T7 RNA polymerase promoter was introduced to the 5'end or 3'end of the corresponding fragment, and 2 subsequent PCR reactions were performed to generate sense and antisense RNA strands. Sense and antisense strands were pooled together, purified by phenol-chloroform extraction, and resuspended in $16 \mu$ l of water before being annealed by incubating at $72^{\circ} \mathrm{C}$ then $37^{\circ} \mathrm{C}$ and finally on ice. dsRNA was mixed with $80 \mu \mathrm{l}$ of homogenized calf liver and $2 \mu \mathrm{l}$ of red food coloring to assess food intake. Planarians were starved

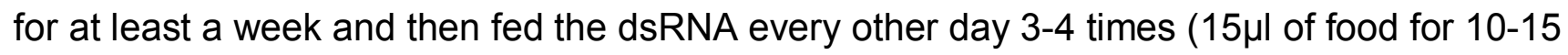
animals). Animals that did not feed were discarded. For behavioral experiments animals were used between one day and four days after last feeding, phototaxis was used at the end of each experiment to ensure viability.

\section{Expression analysis by Q-PCR}

Total RNA from UNC22, Smed-AP2 and Smed-TRPA1 knockdown planarians was purified using Trizol reagent (Life Technologies). First strand cDNA was synthesized using MultiScribe Reverse 
Transcriptase (Fisher Scientific) from DNAse-treated (TURBO DNAse, Ambion) total RNA. qPCR reactions were performed using the EvaGreen dye (Biotium). 4 biological replicates were run for each treatment, with clathrin mRNA detected as reference gene for quantifying expression changes using the delta-delta Ct method and normalizing to the expression obtained in the control RNAi treatment. Smed-TRPA1 was detected using the primers 5'-ACTCTCATCAACAGACAGACTTGT-3' and 5'ATTTCAGCCTCTGGATCCATTTCC-3' and clathrin primers were 5'-GACTGCGGGCTTCTATTGAG3' and 5'-GCGGCAATTCTTCTGAACTC-3'. Results were compared using Kruskal-Wallis one-way ANOVA.

\section{Fluorescent in situ hybridization (FISH)}

Smed-TRPA1 riboprobes were generated from a PCR fragment flanked by T7 promoter sequences using RNA DIG-labeling mix (Sigma-Aldrich). After in vitro transcription, antisense probes were precipitated with $100 \%$ ethanol and resuspended in $25 \mu \mathrm{L}$ of deionized formamide. Planarians were killed in $5 \% \mathrm{~N}$-Acetyl cysteine, fixed in $4 \%$ formaldehyde, followed by dehydration and overnight bleaching in $6 \% \mathrm{H}_{2} \mathrm{O}_{2}$ on a light box. Animals were preserved in $100 \%$ methanol and stored at $-20^{\circ} \mathrm{C}$. For FISH, planarians were re-hydrated with a methanol:PBST (PBS, $0.1 \%$ triton X-100) dilution series; next, animals were treated with $10 \mathrm{mg} / \mathrm{ml}$ proteinase $\mathrm{K}$, post-fixed in $4 \%$ formaldehyde, and incubated at $56^{\circ} \mathrm{C}$ for 2 hours in pre-hybridization solution ( $50 \%$ of de-ionized formamide, $5 x$ SSC, 0.1 $\mathrm{mg} / \mathrm{ml}$ yeast RNA, 1\% Tween-20 in DEPC-treated water). Hybridization with riboprobes was conducted for $16 \mathrm{~h}$ in hybridization solution (same as pre-hybridization solution plus $5 \%$ dextran sulfate). Then animals were washed in pre-hybridization solution, and then subjected to a dilution series of $2 X$ SSC, then $0.2 X$ SSC, and finally TNTx $(0.1 \mathrm{M}$ Tris $\mathrm{pH} 7.5,0.15 \mathrm{M} \mathrm{NaCl}, 0.3 \%$ Triton X100). Animals were blocked in TNTx plus 5\% horse serum and 5\% Western Blocking Reagent (Sigma-Aldrich) for $2 \mathrm{~h}$ at RT, and then labeled with a sheep anti-DIG-POD antibody (1:2000, SigmaAldrich) in blocking solution overnight at $4^{\circ} \mathrm{C}$. Animals were washed $8 \mathrm{X}$ in TNTx, incubated in 
Tyramide solution with rhodamine (1:500) and $\mathrm{H}_{2} \mathrm{O}_{2}$ for 10 minutes with shaking. Finally, animals were rinsed 6X in TNTx. ISH experiments were performed four times with similar results. To quantify TRPA1+ cells in various groups (Figure 1F-K), ten worms per treatment were imaged with a Leica DM 2500 confocal microscope with a 10X objective and 1.5 digital zoom, and Z-stacks encompassing the thickness of each animal were acquired at $5 \mu \mathrm{m}$ intervals, using constant laser and PMT settings. Zstacks were analyzed using ImageJ: Brightness/Contrast was adjusted in batch using identical settings and max projection images through the animal were produced. From these, the number of fluorescent cells in a defined $\mathrm{ROI}$ in the brain region and a defined $\mathrm{ROI}$ at the periphery (each of constant size, shown as yellow boxes in Figure 1) were counted. The number of fluorescent cells was then plotted and compared by unpaired t-test.

\section{Planarian behavioral assays: heat avoidance}

Heat avoidance was measured in the "Planariometer" (see Supplementary Figure 1). The Planariometer consisted in four independent tiles covered by thin anodized aluminum foil. A hydrophobic ink pen (Super PAP pen - ThermoFisher) was used to create a circular barrier (55mm of diameter) to allow a thin film of water $(1-2 \mathrm{~mm})$ to form a central pool in which the worms can move freely. In each experiment, 2 opposite tiles were set at $32^{\circ} \mathrm{C}$ and 2 at $24^{\circ} \mathrm{C}$ and animal movement was recorded for 4 minutes. The spatial configuration of hot and cool tiles was then reversed for 4 additional minutes (and a second movie acquired) to control for potential spatial biases. Experiments were conducted in the dark with infrared (IR) LED illumination and videos were recorded with an IRsensitive CCD camera (Basler). Five independent groups of 10 animals per treatment were used. The heat avoidance index $\left(\mathrm{Al}=\#\right.$ worms at $24^{\circ} \mathrm{C}-\#$ worms at $32^{\circ} \mathrm{C} /$ total \#worms $)$ was calculated from the last 120 frames of each video (last minute of the video) by measuring the positions of the worms every 3 frames using a custom-made Matlab script. Avoidance index values were compared using Kruskal-Wallis ANOVA followed by Tukey's honest significant difference test. 


\section{Planarian behavioral assays: AITC avoidance}

Five independent groups of 10 animals per treatment were tested in an arena composed of two circular chambers connected by a narrow corridor. At the beginning of the experiment, all animals were placed in chamber 1 together with a small block of control agar ( $1 \%$ agarose dissolved in $1 \mathrm{x}$ APW) or AITC laced agar. The AITC laced agar was made with $1 \%$ agarose in $1 \times$ APW and $50 \mathrm{mM}$ AITC. The chamber was placed in the dark, animals where illuminated with IR light and their behavior was recorded for 5 minutes with a IR-sensitive CCD camera. Videos were analyzed and the number of planarians in each chamber was quantified every 10 frames for the last 125 frames of the video (last minute). The fraction of animals in each chamber was counted after each treatment and compared using Kruskal-Wallis ANOVA followed by Tukey's honest significant difference test.

\section{Planarian behavioral assays: Negative phototaxis}

Four independent groups of 10 worms per treatment were placed in chamber 1 of the arena as described above (AITC avoidance experiments). The arena was either kept completely dark (control condition), alternatively, chamber 1 was exposed to bright light while chamber 2 was kept dark. Animals were allowed to distribute for 2 minutes before the number of worms in each chamber was counted.

\section{$\underline{\text { Cell transfections }}$}

pAC-GFP, pAC-Smed-TRPA1 and pAC-dTRPA1-A were generated by cloning GFP, Smed-TRPA1 ORF (see above) and a dTRPA1-A cDNA (a gift of Dan Tracey) into pCR ${ }^{\mathrm{TM}}$ 8/GW/TOPO® TA (ThermoFisher) and then transferring them into pAC-GW expression vector [created by ligating the Gateway cassette from pMartini Gate C R2-R1 (Addgene plasmid \#36436) cut with Xhol and Xbal into pAc5.1/V5-His A (ThermoFisher)]. S2R+ cells (a gift from R. Carthew) were cultured in 
Schneider's Drosophila Medium (Lonza) supplemented with $10 \%$ fetal bovine serum and $1 \%$

penicillin-Streptomycin mixture (100units $/ \mathrm{mL}$ and $100 \mu \mathrm{g} / \mathrm{mL}$ respectively, Fisher Scientific). For

electrophysiological recordings, S2R+ cells where grown on coverslips in Schneider's Drosophila Medium supplemented with $50 \mu \mathrm{M} \mathrm{LaCl} 3$ and transfected with $50 \mathrm{ng}$ of pAC-GFP vector and $500 \mathrm{ng}$ of either PAC-dTRPA1-A or pAC-Smed-TRPA1 vectors mixed with $4 \mu$ l of enhancer and $150 \mu$ l of buffer EC. After 5 min, $6.5 \mu$ of Effectene ${ }^{\circledR}$ Transfection Reagent (Qiagen) was added and the mix was incubated for $10 \mathrm{~min}$ before being dispensed to the cells. Transfected cells were incubated at RT for at least $36 \mathrm{~h}$ to allow gene expression.

\section{Electrophysiological recordings}

Whole cell voltage clamp was performed on S2R+ transfected cells identified by GFP fluorescence. The intracellular solution contained: $140 \mathrm{mM}$ methanesulfonic acid, $2 \mathrm{mM} \mathrm{MgCl}_{2}, 1 \mathrm{mM}$ EGTA, $5 \mathrm{mM}$ HEPES, $1 \mathrm{mM} \mathrm{Na}{ }_{2}$ ATP; $p H$ was adjusted to 7.3 with $\mathrm{CsOH}$ and the osmolarity was adjusted to $315 \pm$ $5 \mathrm{mOsml}$ with sucrose. The extracellular solution contained: $140 \mathrm{mM} \mathrm{NaCl}, 5 \mathrm{mM} \mathrm{KCl}, 1 \mathrm{mM} \mathrm{CaCl}_{2}$, $1 \mathrm{mM}$ HEPES, $10 \mathrm{mM}$ Glucose; $\mathrm{pH}$ was adjusted to 7.2 with $\mathrm{NaOH}$ and the osmolarity was adjusted to $310 \pm 5 \mathrm{mOsml}$ with sucrose. Patch pipettes resistance ranged from 5 to $10 \mathrm{M} \Omega$. Recordings were obtained with an AxoPatch 200b amplifier (Axon Instruments), and analyzed with AxoGraph software and custom-made Matlab scripts. Recordings were made with $1 \times$ output gain and $5 \mathrm{KHz}$ low pass filter. Bath offset and capacitance were compensated; series resistance was $9.5 \pm 5.5 \mathrm{M} \Omega$ without compensation. Recordings were made at RT $\left(22-23^{\circ} \mathrm{C}\right)$ and temperature stimulation was achieved by raising the temperature of the bath solution via an inline heater (HPT-2A, ALA Scientific Instruments) and a TC-20 temperature controller (NPI Electronics). Temperature was monitored with a T-384 thermocouple (Physitemp Instruments) tethered to the electrode holder, so that the tip of the thermocouple was approximately at a constant distance from the tip of the recording electrode (1-2 $\mathrm{mm})$. Chemical stimulation was achieved by bath perfusion of extracellular solution containing $500 \mu \mathrm{M}$ 
of allyl isothiocyanate (AITC, Sigma). Cells were held at $-60 \mathrm{mV}$ and currents were monitored during heat and chemical stimulation. Current-voltage relationships were constructed by averaging three step protocols consisting of $100 \mathrm{~ms}$ steps of $20 \mathrm{mV}$ from -100 to $100 \mathrm{mV}$ separated by $400 \mathrm{~ms}$. These IV relationships where made at $\mathrm{RT}$, during the heat stimulation, and at the end of a $3 \mathrm{~min}$ AITC application. Note that Smed-TRPA1 did not appear to respond to cooling. For the AITC and Hydrogen peroxide dose responses $\left(\mathrm{H}_{2} \mathrm{O}_{2}\right.$, Sigma, $\left.30 \% \mathrm{w} / \mathrm{w}\right)$ we used 1 min stimulation at each concentration. Recordings were performed as described above with the exception that the intracellular solution contained 140mM K-gluconate instead of Cesium.

\section{Fly strains and transgenes}

Flies were reared on standard cornmeal agar medium at room temperature (RT). The following fly strains were used: Canton-special, isogenic $\mathrm{w}^{1118}$ (a gift from Marcus C. Stensmyr); elav-Gal4/CyO; $\operatorname{trpA}^{1}{ }^{1}$ (BDSC\#26504, backcrossed 5 times); 5xUAS-TRPA1-C (a gift from D. Tracey); tub-cytoroGFP2-Orp1 (ref \#50). To generate UAS-SmedTRPA1 flies, Smed-TRPA1 cDNA (see above) was cloned into $\mathrm{pCR}^{\mathrm{TM}} 8 / \mathrm{GW} / \mathrm{TOPO}{ }^{\mathrm{T}} \mathrm{TA}$ (Invitrogen) and then transferred into a 40xUAS destination vector created introducing the Gateway ${ }^{\circledR}$ cassette into pJFRC8-40XUAS- IVS-mCD8::GFP (Addgene \#26221) via the Xhol/ Xbal restriction sites. This construct was then used for embryo injection by BestGene Inc. to generate P[40XUAS::Smed-TRPA1]attP40 flies. Similarly, UAS-humanTRPA1 flies were obtained starting from a human TRPA1 cDNA (NP_015628; a gift from Mark Hoon) to generate P[40XUAS::hTRPA1]attP40 flies. Expression of the transgenes was confirmed by RT PCR. Full genotypes of fly stocks used in Figure $5: w^{1118} . w^{1118} ; \operatorname{TRPA}^{1} . w^{1118}$; elav-Gal4/+; TRPA $1^{1} / \operatorname{TRPA}^{1}$. $w^{1118} ;+/ U A S-T R P A 1-C ; T_{R P A} 1^{1} / T R P A 1^{1} . w^{1118}$; elav-Gal4/UAS-TRPA1-C; TRPA $1^{1} /$ TRPA $^{1} . w^{1118}$; +/UAS-Smed-TRPA1; TRPA $1^{1} /$ TRPA $^{1}$. $w^{1118}$; elav-Gal4/UAS-Smed-TRPA1; TRPA1 ${ }^{1} /$ TRPA1 $^{1} . w^{1118}$; +/UAS-human TRPA1; TRPA $1^{1} /$ TRPA $^{1}{ }^{1} . w^{1118}$; elav-Gal4/UAS-human TRPA1; TRPA $1^{1} /$ TRPA $^{1}$. 


\section{Drosophila behavioral assays}

Temperature preference assay were performed as previously described (Gallio et al., 2011). In brief, Avoidance Index (Al) values for the test temperatures (TT) are calculated as follows: $\mathrm{Al}=\#$ flies at $25^{\circ} \mathrm{C}$ - \# flies at TT/(total \# flies). Al values were compared using ANOVA or 2-way ANOVA as previously described. Avoidance index values for experiments with Gal4 and UAS lines were compared by two-way ANOVA (threshold $\mathrm{P}=0.01$ ). Kolmogorov Smirnov tests were used to test for a normally distributed sample. Homogeneity of variance for each data set was confirmed by calculating the Spearman correlation between the absolute values of the residual errors and the observed values of the dependent variable (threshold $P=0.05$ ). Statistical analysis was carried out in MATLAB; sample sizes were chosen to reliably measure experimental parameters. Experiments did not involve randomization or blinding. All temperature preference experiments were performed in a custom chamber kept at a constant $\mathrm{RH}$ of $40 \%$. Heat and AITC-vapor incapacitation experiments (Figure 4) were performed as follows: Flies of the genotypes elav/+, UAS-Smed-TRPA1/+, UAS-TRPA1-1/+ (negative controls), elav-Gal4>UAS-TRPA1-A (positive control) and elav-Gal4>UAS-SmedTRPA1(experimental animals), were used to test responses to temperature and AITC. For heat incapacitation, groups of 10 flies were collected in empty vials and placed in a $25^{\circ} \mathrm{C}$ incubator for at least an hour before the experiments. Next, the vials were submerged in water (pre-heated to $35^{\circ} \mathrm{C}$ ) and kept submerged until the internal air temperature of the tube had been at $35^{\circ} \mathrm{C}$ for one minute (as measured by a thermocouple). Following this, the number of incapacitated flies (i.e. flies that had dropped to the bottom of the tube) was counted. Vials were then placed at RT for three additional minutes, and the number of incapacitated flies scored every minute to measure recovery. For the exposure to AITC vapors, groups of 10 flies of each genotype (see above), were collected in $15 \mathrm{~mL}$ tubes for bacterial culture with small holes to allow air flow. These $15 \mathrm{~mL}$ culture tube were placed inside a $50 \mathrm{~mL}$ conical tube containing a small piece of filter paper with $1 \mu \mathrm{l}$ of $2.5 \mathrm{M}$ AITC. Flies were exposed to AITC vapors for 10 minutes and then transferred to clean vials for recovery. The number 
of incapacitated animals was recorded every minute during AITC exposure and every 5 minutes during recovery. For the pro-oxidant feeding experiments, groups of twenty 3-5 day-old flies of the appropriate genotype were starved for 18 hours in vials with a Kim-wipe saturated by water. Flies were then fed for three hours on Nutri-Fly ${ }^{\mathrm{TM}}$ Instant Medium (Genesee Scientific \#66-117) prepared with the respective pro-oxidant solution at a ratio of medium to liquid of 1:3. The liquid used contained the pro-oxidant and sucrose, or sucrose alone ('mock'). Final concentrations: all samples $=2 \%$ sucrose; $\mathrm{H}_{2} \mathrm{O}_{2}=5 \%$, Paraquat (Sigma \#856177) $=50 \mathrm{mM}$. Immediately after, the animals were tested for temperature preference as described above. Food intake was monitored in parallel experiments using green food colorant $(25 \mu \mathrm{l}$ for $3 \mathrm{ml}$ of solution).

\section{$\underline{\text { ROS imaging }}$}

To evaluate ROS levels in response to heat stimuli in intact live planarians and Drosophila tissues, we used the fluorogenic oxidative stress indicator carboxy-H2DCFDA (Molecular Probes \#I36007) per the manufacturer's guidelines (and see below). ROS levels were imaged on an LSM510 Zeiss confocal microscope equipped using a 488 argon laser. Temperature stimuli were generated with a Model 5000 KT stage controller (20/20 Technology) and the temperature was recorded using NI USBTC01 equipped with a thermocouple probe (National Instruments). Intact planarians were incubated for an hour in $25 \mu \mathrm{M}$ carboxy-H2DCFDA (diluted in APW) and washed briefly in APW prior to imaging.

To minimize movements during scanning, animals were placed into a tight-fitting custom made Sylgard frame mounted on a glass slide and filled with APW. The preparation was sealed with a cover slip. For real time ROS detection, planarians were imaged continuously with a 5x/0.16 Zeiss air objective at $256 \times 256$ pixel resolution and $2 \times$ optical zoom at $0.395 \mathrm{msec}$ frame rate during a heat ramp of $\Delta \mathrm{t}=20>35^{\circ} \mathrm{C}$, speed $=\sim 1^{\circ} \mathrm{C} / 4$ seconds. ROS induced fluorescence was measured from confocal images acquired at low resolution and using a fully open pinhole, from ROIs corresponding to large parts of the head region $(512 \times 512$ pixel resolution $1 \times$ optical zoom with a $5 \times$ Zeiss air 
objective). Drosophila salivary glands were dissected in PBS and incubated with $25 \mu \mathrm{M}$ carboxy-

H2DCFDA (diluted in PBS) for an hour at room temperature prior to heat stimulation. Tissues were then briefly washed in PBS and transferred into a custom made thin Sylgard frame containing PBS and mounted on a glass slide. The preparation was sealed with a cover slip. For real time ROS detection in Drosophila, salivary glands were imaged continuously with a 10xZeiss air objective at $256 \times 256$ pixel resolution and $2 x$ optical zoom at $0.395 \mathrm{msec}$ frame rate during a heat ramp of $\Delta \mathrm{t}=20>45^{\circ} \mathrm{C}$, speed $=\sim 1^{\circ} \mathrm{C} / 4$ seconds. $\mathrm{DF} / \mathrm{F}$ analysis was carried out using custom scripts in MATLAB, base fluorescence was calculated using all frames preceding temperature trigger (occurring at $30 \mathrm{~s})$. Confocal stacks of about $100 \mu \mathrm{m}$ at $5 \mu \mathrm{m}$ steps were obtained at $512 \times 512$ pixel resolution $1 \mathrm{x}$ optical zoom with a 10x Zeiss air objective.

For in vivo $\mathrm{H} 2 \mathrm{O} 2$ detection in intact animals, tub-cyto-roGFP2-Orp1 larvae were placed on a heated surface set to $\sim 35^{\circ} \mathrm{C}$ for 5 seconds. As a positive control, larvae were exposed to $25 \mu \mathrm{M} \mathrm{H} 2 \mathrm{O} 2$ for 10 minutes. Animals were rapidly dissected post treatment in PBS to extract their wing imaginal discs (as described in ref \#50). The tissues were mounted in glycerol and immediately imaged on a Leica SP5 inverted confocal microscope equipped with a 405 UV and a 488 Argon laser and a 10x air objective at $512 \times 512$ pixel resolution and $400 \mathrm{~Hz}$. Image acquisition and processing were performed as above. We used two-sample T-test to calculate significant difference $(p<0.05)$ between treatments and controls. Excitation of the biosensor fluorescence by the $405 \mathrm{~nm}$ and $488 \mathrm{~nm}$ laser lines was performed sequentially and stack by stack. Emission was detected at 500-570 nm. Image processing was performed with imageJ. Control fluorescence (i.e. of untreated tissue) was set to 1. 


\section{References:}

1 Rosenzweig, M. et al. The Drosophila ortholog of vertebrate TRPA1 regulates thermotaxis. Genes \& development 19, 419-424, doi:10.1101/gad.1278205 (2005).

2 Neely, G. G. et al. TrpA1 regulates thermal nociception in Drosophila. PloS one 6, e24343, doi:10.1371/journal.pone.0024343 (2011).

3 Zhong, L. et al. Thermosensory and nonthermosensory isoforms of Drosophila melanogaster TRPA1 reveal heat-sensor domains of a thermoTRP Channel. Cell reports 1, 43-55, doi:10.1016/j.celrep.2011.11.002 (2012).

Kremeyer, B. et al. A gain-of-function mutation in TRPA1 causes familial episodic pain syndrome. Neuron 66, 671-680, doi:10.1016/j.neuron.2010.04.030 (2010).

Story, G. M. et al. ANKTM1, a TRP-like Channel Expressed in Nociceptive Neurons, Is Activated by Cold Temperatures. Cell 112, 819-829, doi:http://dx.doi.org/10.1016/S0092-8674(03)00158-2 (2003).

Jordt, S. E. et al. Mustard oils and cannabinoids excite sensory nerve fibres through the TRP channel ANKTM1. Nature 427, 260-265, doi:10.1038/nature02282 (2004).

Bandell, M. et al. Noxious cold ion channel TRPA1 is activated by pungent compounds and bradykinin. Neuron 41, 849-857 (2004).

8

Macpherson, L. J. et al. The pungency of garlic: activation of TRPA1 and TRPV1 in response to allicin. Current biology : $C B$ 15, 929-934, doi:10.1016/j.cub.2005.04.018 (2005).

9

Bautista, D. M. et al. Pungent products from garlic activate the sensory ion channel TRPA1. Proceedings of the National Academy of Sciences of the United States of America 102, 12248-12252, doi:10.1073/pnas.0505356102 (2005).

11 Macpherson, L. J. et al. An ion channel essential for sensing chemical damage. The Journal of neuroscience : the official journal of the Society for Neuroscience 27, 11412-11415, doi:10.1523/JNEUROSCI.3600-07.2007 (2007).

12 Viswanath, V. et al. Opposite thermosensor in fruitfly and mouse. Nature 423, 822-823, doi:10.1038/423822a (2003).

13 Kang, K. et al. Analysis of Drosophila TRPA1 reveals an ancient origin for human chemical nociception. Nature 464, 597-600, doi:10.1038/nature08848 (2010).

14 Chatzigeorgiou, M. et al. Specific roles for DEG/ENaC and TRP channels in touch and thermosensation in C. elegans nociceptors. Nature neuroscience 13, 861-868, doi:10.1038/nn.2581 (2010).

15 Laursen, W. J., Bagriantsev, S. N. \& Gracheva, E. O. TRPA1 channels: chemical and temperature sensitivity. Current topics in membranes 74, 89-112, doi:10.1016/B978-0-12-800181-3.00004-X (2014).

16 Chen, J. et al. Species differences and molecular determinant of TRPA1 cold sensitivity. Nature communications 4, 2501, doi:10.1038/ncomms3501 (2013).

17 Saito, S. et al. Heat and noxious chemical sensor, chicken TRPA1, as a target of bird repellents and identification of its structural determinants by multispecies functional comparison. Molecular biology and evolution 31, 708-722, doi:10.1093/molbev/msu001 (2014).

18 Gracheva, E. O. et al. Molecular basis of infrared detection by snakes. Nature 464, 1006-1011, doi:10.1038/nature08943 (2010). 
19 Saito, S. et al. Analysis of transient receptor potential ankyrin 1 (TRPA1) in frogs and lizards illuminates both nociceptive heat and chemical sensitivities and coexpression with TRP vanilloid 1 (TRPV1) in ancestral vertebrates. The Journal of biological chemistry 287, 30743-30754, doi:10.1074/jbc.M112.362194 (2012).

20 Oda, M., Kurogi, M., Kubo, Y. \& Saitoh, O. Sensitivities of Two Zebrafish TRPA1 Paralogs to Chemical and Thermal Stimuli Analyzed in Heterologous Expression Systems. Chemical senses 41, 261-272, doi:10.1093/chemse/bjv091 (2016).

21 Prober, D. A. et al. Zebrafish TRPA1 channels are required for chemosensation but not for thermosensation or mechanosensory hair cell function. The Journal of neuroscience : the official journal of the Society for Neuroscience 28, 10102-10110, doi:10.1523/JNEUROSCI.2740-08.2008 (2008).

22 Kohno, K., Sokabe, T., Tominaga, M. \& Kadowaki, T. Honey bee thermal/chemical sensor, AmHsTRPA, reveals neofunctionalization and loss of transient receptor potential channel genes. The Journal of neuroscience : the official journal of the Society for Neuroscience 30, 12219-12229, doi:10.1523/JNEUROSCI.2001-10.2010 (2010).

23 Wang, G. et al. Anopheles gambiae TRPA1 is a heat-activated channel expressed in thermosensitive sensilla of female antennae. The European journal of neuroscience 30, 967-974, doi:10.1111/j.14609568.2009.06901.x (2009).

24 Kang, K. et al. Modulation of TRPA1 thermal sensitivity enables sensory discrimination in Drosophila. Nature 481, 76-80, doi:10.1038/nature10715 (2012).

25 Dunn, C. W., Giribet, G., Edgecombe, G. D. \& Hejnol, A. Animal Phylogeny and Its Evolutionary Implications. Annual Review of Ecology, Evolution, and Systematics 45, 371-395, doi:doi:10.1146/annurev-ecolsys-120213-091627 (2014).

26 Newmark, P. A., Reddien, P. W., Cebria, F. \& Sanchez Alvarado, A. Ingestion of bacterially expressed double-stranded RNA inhibits gene expression in planarians. Proceedings of the National Academy of Sciences of the United States of America 100 Suppl 1, 11861-11865, doi:10.1073/pnas. 1834205100 (2003).

27 Wenemoser, D., Lapan, S. W., Wilkinson, A. W., Bell, G. W. \& Reddien, P. W. A molecular wound response program associated with regeneration initiation in planarians. Genes \& development 26, 9881002, doi:10.1101/gad.187377.112 (2012).

28 Gallio, M., Ofstad, T. A., Macpherson, L. J., Wang, J. W. \& Zuker, C. S. The coding of temperature in the Drosophila brain. Cell 144, 614-624, doi:10.1016/j.cell.2011.01.028 (2011).

29 Harrath, A. H., Charni, M., Sluys, R., Zghal, F. \& Tekaya, S. Ecology and distribution of the freshwater planarian Schmidtea mediterranea in Tunisia. Italian Journal of Zoology 71, 233-236, doi:10.1080/11250000409356577 (2004).

30 Inoue, T., Yamashita, T. \& Agata, K. Thermosensory signaling by TRPM is processed by brain serotonergic neurons to produce planarian thermotaxis. The Journal of neuroscience : the official journal of the Society for Neuroscience 34, 15701-15714, doi:10.1523/JNEUROSCI.5379-13.2014 (2014).

31 Wang, H., Schupp, M., Zurborg, S. \& Heppenstall, P. A. Residues in the pore region of Drosophila transient receptor potential A1 dictate sensitivity to thermal stimuli. The Journal of physiology 591, 185201, doi:10.1113/jphysiol.2012.242842 (2013).

32 Cordero-Morales, J. F., Gracheva, E. O. \& Julius, D. Cytoplasmic ankyrin repeats of transient receptor potential A1 (TRPA1) dictate sensitivity to thermal and chemical stimuli. Proceedings of the National 
bioRxiv preprint doi: https://doi.org/10.1101/185405; this version posted September 12, 2017. The copyright holder for this preprint (which was not certified by peer review) is the author/funder, who has granted bioRxiv a license to display the preprint in perpetuity. It is made available under aCC-BY-NC-ND 4.0 International license.

Academy of Sciences of the United States of America 108, E1184-1191, doi:10.1073/pnas.1114124108 (2011).

33 Kwon, Y., Shim, H.-S., Wang, X. \& Montell, C. Control of thermotactic behavior via coupling of a TRP channel to a phospholipase C signaling cascade. Nature neuroscience 11, 871-873, doi:http://www.nature.com/neuro/journal/v11/n8/suppinfo/nn.2170_S1.html (2008).

34 Lee, Y. \& Montell, C. Drosophila TRPA1 functions in temperature control of circadian rhythm in pacemaker neurons. The Journal of neuroscience : the official journal of the Society for Neuroscience 33, 6716-6725, doi:10.1523/JNEUROSCI.4237-12.2013 (2013).

$35 \mathrm{Ni}$, L. et al. A gustatory receptor paralogue controls rapid warmth avoidance in Drosophila. Nature 500, 580-584, doi:10.1038/nature12390 (2013).

36 Niethammer, P., Grabher, C., Look, A. T. \& Mitchison, T. J. A tissue-scale gradient of hydrogen peroxide mediates rapid wound detection in zebrafish. Nature 459, 996-999, doi:http://www.nature.com/nature/journal/v459/n7249/suppinfo/nature08119_S1.html (2009).

37 Moreira, S., Stramer, B., Evans, I., Wood, W. \& Martin, P. Prioritization of Competing Damage and Developmental Signals by Migrating Macrophages in the Drosophila Embryo. Current Biology 20, 464470, doi:10.1016/j.cub.2010.01.047 (2010).

38 Pirotte, N. et al. Reactive Oxygen Species in Planarian Regeneration: An Upstream Necessity for Correct Patterning and Brain Formation. Oxidative medicine and cellular longevity 2015, 392476, doi:10.1155/2015/392476 (2015).

39 Andersson, D. A., Gentry, C., Moss, S. \& Bevan, S. Transient receptor potential A1 is a sensory receptor for multiple products of oxidative stress. The Journal of neuroscience : the official journal of the Society for Neuroscience 28, 2485-2494, doi:10.1523/jneurosci.5369-07.2008 (2008).

40 Bessac, B. F. et al. TRPA1 is a major oxidant sensor in murine airway sensory neurons. The Journal of clinical investigation 118, 1899-1910, doi:10.1172/JCI34192 (2008).

41 Du, E. J. et al. Nucleophile sensitivity of Drosophila TRPA1 underlies light-induced feeding deterrence. eLife 5, doi:10.7554/eLife.18425 (2016).

42 Guntur, A. R. et al. H2O2-Sensitive Isoforms of Drosophila melanogaster TRPA1 Act in Bitter-Sensing Gustatory Neurons to Promote Avoidance of UV During Egg-Laying. Genetics 205, 749-759, doi:10.1534/genetics.116.195172 (2017).

43 Guntur, A. R. et al. Drosophila TRPA1 isoforms detect UV light via photochemical production of H2O2. Proceedings of the National Academy of Sciences of the United States of America 112, E57535761, doi:10.1073/pnas.1514862112 (2015).

44 Xu, C., Luo, J., He, L., Montell, C. \& Perrimon, N. Oxidative stress induces stem cell proliferation via TRPA1/RyR-mediated Ca2+ signaling in the Drosophila midgut. eLife 6, doi:10.7554/eLife.22441 (2017).

45 Kim, M. J. \& Johnson, W. A. ROS-mediated activation of Drosophila larval nociceptor neurons by UVC irradiation. BMC neuroscience 15, 14, doi:10.1186/1471-2202-15-14 (2014).

46 Birkholz, T. R. \& Beane, W. S. The planarian TRPA1 homolog mediates extraocular behavioral responses to near ultraviolet light. The Journal of experimental biology, doi:10.1242/jeb.152298 (2017).

47 Miyake, T. et al. Cold sensitivity of TRPA1 is unveiled by the prolyl hydroxylation blockade-induced sensitization to ROS. Nature communications 7, 12840, doi:10.1038/ncomms 12840 (2016). 
48 Kalyanaraman, B. et al. Measuring reactive oxygen and nitrogen species with fluorescent probes: challenges and limitations. Free radical biology \& medicine 52, 1-6, doi:10.1016/j. freeradbiomed.2011.09.030 (2012).

49 Gutscher, M. et al. Proximity-based protein thiol oxidation by H2O2-scavenging peroxidases. The Journal of biological chemistry 284, 31532-31540, doi:10.1074/jbc.M109.059246 (2009).

50 Albrecht, S. C., Barata, A. G., Grosshans, J., Teleman, A. A. \& Dick, T. P. In vivo mapping of hydrogen peroxide and oxidized glutathione reveals chemical and regional specificity of redox homeostasis. Cell metabolism 14, 819-829, doi:10.1016/j.cmet.2011.10.010 (2011).

51 Bayne, A. C., Mockett, R. J., Orr, W. C. \& Sohal, R. S. Enhanced catabolism of mitochondrial superoxide/hydrogen peroxide and aging in transgenic Drosophila. The Biochemical journal 391, 277284, doi:10.1042/BJ20041872 (2005).

52 Rzezniczak, T. Z., Douglas, L. A., Watterson, J. H. \& Merritt, T. J. Paraquat administration in Drosophila for use in metabolic studies of oxidative stress. Analytical biochemistry 419, 345-347, doi:10.1016/j.ab.2011.08.023 (2011).

53 Inoue, T., Hoshino, H., Yamashita, T., Shimoyama, S. \& Agata, K. Planarian shows decision-making behavior in response to multiple stimuli by integrative brain function. Zoological letters 1, 7, doi:10.1186/s40851-014-0010-z (2015).

54 Umesono, Y., Tasaki, J., Nishimura, K., Inoue, T. \& Agata, K. Regeneration in an evolutionarily primitive brain--the planarian Dugesia japonica model. The European journal of neuroscience 34, 863869, doi:10.1111/j.1460-9568.2011.07819.x (2011). 


\section{Supplementary Information Line}

Supplementary information is linked to the on-line version of the paper at www.nature.com

\section{Acknowledgments}

We thank D. Tracey and R. Carthew for reagents. Andrew Kuang and Leah Vinson for technical assistance, Lindsey Macpherson, David Yarmolinsky and members of the Gallio Lab for comments on the manuscript, Indira Raman for technical advice and Marcus Stensmyr for the kind gift of the fly drawing in Fig1. Work in the Gallio lab is supported by NIH grant R01NS086859 (to M.G.), the Chicago Biomedical Consortium with support from the Searle Funds at the Chicago Community Trust (to E.E.Z.), and by training grant 2T32MH067564 (to O.M.A.). Work in the Petersen Lab is supported by an NIH Director's New Innovator award (1DP2DE024365-01).

\section{Author contributions}

M.G. designed the study, analyzed the data, and wrote the paper with critical input from all authors; O.M.A. performed all planarian behavioral experiments and electrophysiology and analyzed the corresponding data. E.E.Z. performed all fly rescue experiments and ROS assays and analyzed the corresponding data. A.P. cloned Smed-TRPA1, produced rescue constructs and transgenics and analyzed sequences with help from C.P.P; A.P. O.M.A and C.V.D. performed Q-PCR and ISH experiments. E.E.Z and O.M.A generated human-TRPA1 expressing flies.

\section{Author information}

Reprints and permissions information is available at www.nature.com/reprints. The authors declare no competing financial interests. Correspondence and requests for materials should be addressed to M.G. (marco.gallio@northwestern.edu). 

under aCC-BY-NC-ND 4.0 International license.

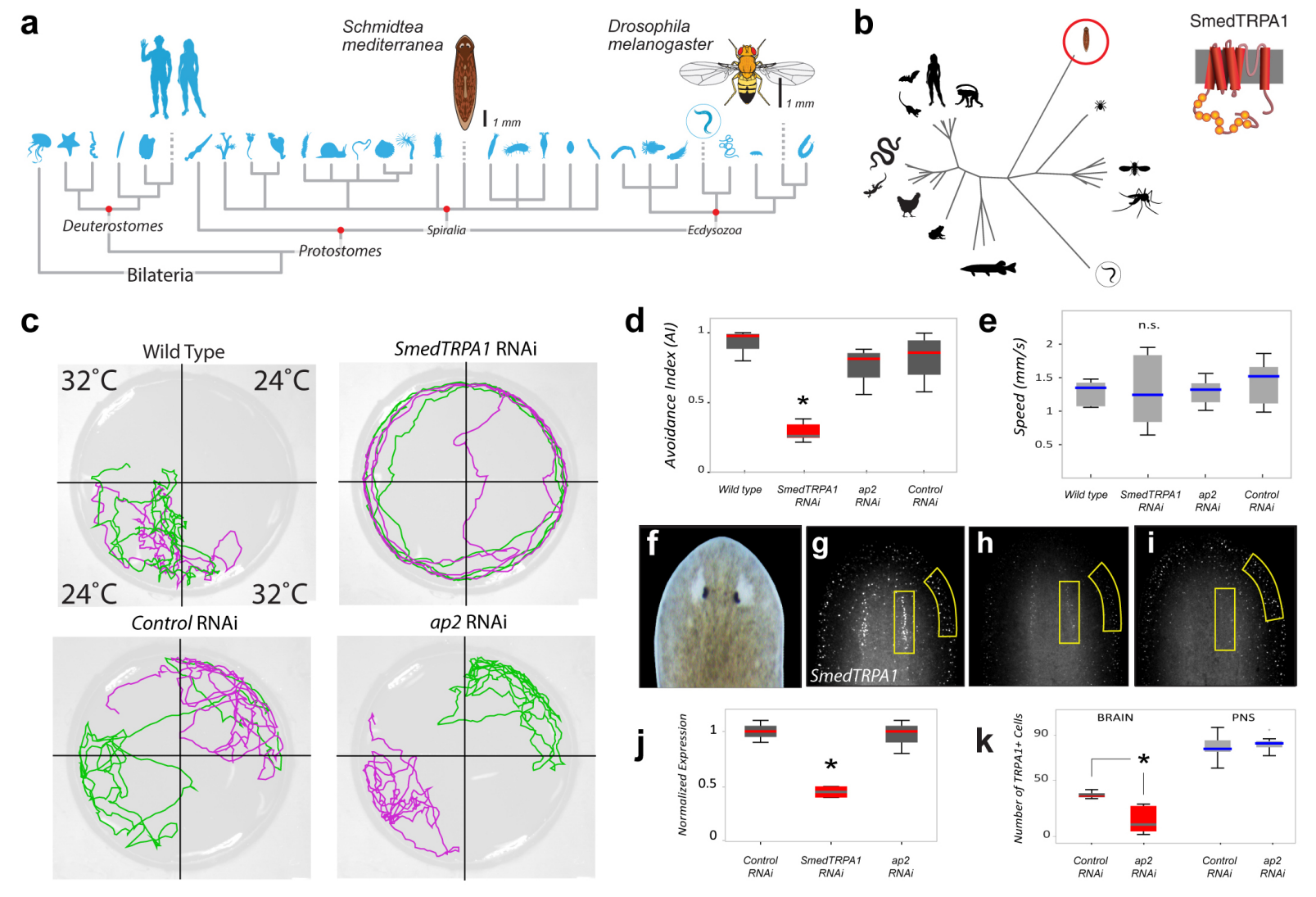

Figure 1: Smed-TRPA1 is required for noxious heat avoidance in the planarian worm $S$.

mediterranea. a) Phylogeny of Bilateria, showing the position of Schmidtea (C. elegans is circled). b) Phylogenetic tree constructed from an alignment of full-length TRPA1 protein sequences from a variety of species, Smed-TRPA1 is circled and a model of the channel's structure is shown (circles=ankyrin repeats, cylinders=transmembrane domains). c) 2-choice assay for heat avoidance. In each trial two opposing floor tiles are set to $24^{\circ} \mathrm{C}$ and two to $32^{\circ} \mathrm{C}$ (noxious heat). Tracks of two worms during one such trial are shown in green and purple. Unlike wild-type, controls (unc22 RNAi), and ap2 RNAi, Smed-TRPA1 RNAi animals were not confined to the cool quadrants. d) Avoidance index for $32^{\circ} \mathrm{C}$ for RNAi animals. Smed-TRPA1 RNAi animals show a reduced avoidance index for heat ( $N=5$ groups of 10 animals, ${ }^{*} P<0.05$, ANOVA). e) Smed-TRPA1 RNAi does not impact the animal's speed of movement ( $\mathrm{N}=10$ animals; $\mathrm{n} . \mathrm{s}$. = not significantly different). $\mathbf{f}-\mathrm{i})$ In situ hybridization with a Smed-TRPA1 probe in (g) Control (unc22) RNAi, (h) Smed-TRPA1 RNAi and (i) ap2 RNAi animals (head region, see $\mathbf{f}$ ), demonstrates overall reduction of mRNA by Smed-TRPA1 RNAi (independent quantification by $\mathrm{Q}-\mathrm{PCR}$ is shown in $\mathbf{j} ; \mathrm{N}=4$ replicates of 3 animals each, ${ }^{*}=\mathrm{P}<0.05$, ANOVA). k) In contrast, ap2 RNAi reduces the number of Smed-TRPA1-expressing cells in the brain region, but not in the periphery ( $\mathrm{N}=9$ animals, ${ }^{*}=\mathrm{P}<0.001$, t-test); in all box plots, edges of the boxes $=$ first and third quartiles, line $=$ median, whiskers $=$ data range, crosses $=$ outliers . 

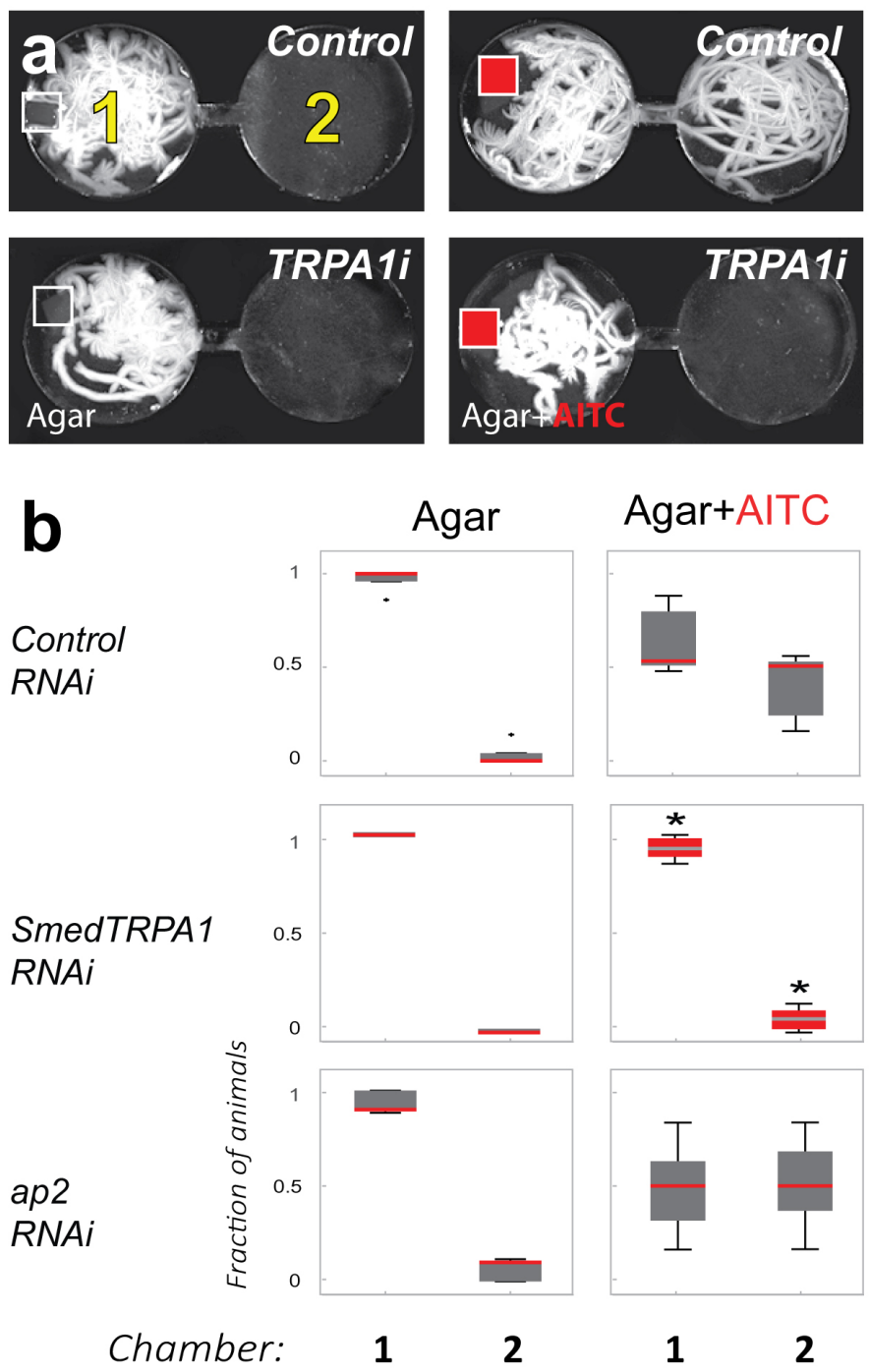

Figure 2: Smed-TRPA1 is required for behavioral avoidance of the irritant chemical AITC. a) Twochamber arena designed to quantify behavioral avoidance of chemical agonists of TRPA1. Planarian worms are introduced in chamber 1 in the presence of a mock Agar pellet (empty squares) or Agar+AITC (50 mM; red squares); their movement is then recorded for 5 minutes. The panels are maximum-projections of 5' movies, illustrating the extent of worm movement (white tracks). b) In the presence of agar alone, control (unc22), ap2 and Smed-TRPA1 RNAi worms do not readily cross the narrow channel connecting chambers 1 and 2. In the presence of AITC, both control (unc22) and ap2 RNAi worms exit chamber 1 and explore chamber 2. In contrast, Smed-TRPA1 RNAi animals overwhelmingly remain in chamber $1(\mathrm{~N}=5$ groups of 10 animals, fraction was calculated on the last 1 ' of video; * $=\mathrm{P}<0.01$, ANOVA comparing fraction of animals in chamber 1 or 2 across treatments; edges of the boxes $=$ first and third quartiles, line $=$ median, whiskers $=$ data range, crosses $=$ outliers). 
a

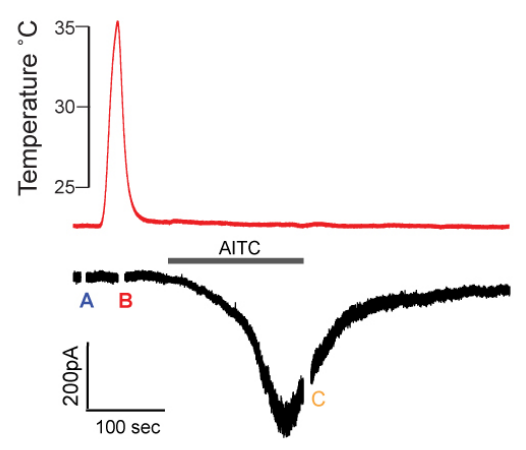

C

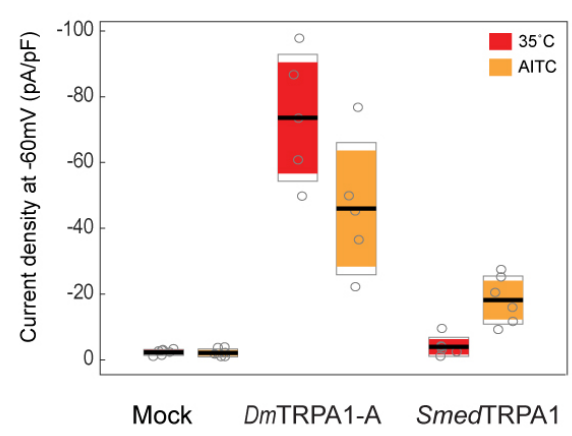

b

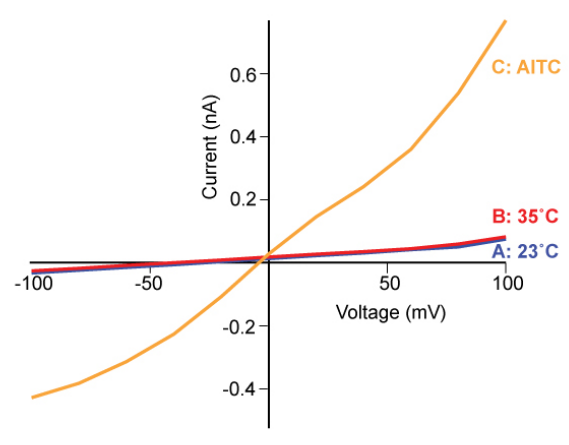

d

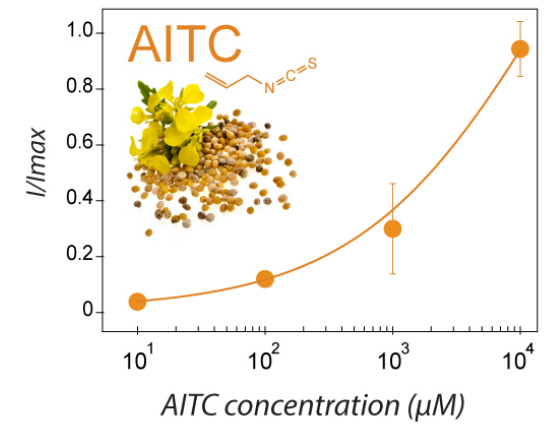

Figure 3: Smed-TRPA1 expressed in Drosophila cells is activated by AITC but not by heat. a) S2R+ Drosophila cells voltage clamped at $-60 \mathrm{mV}$ were stimulated by heat (red trace) and by bath application of AITC $(500 \mu \mathrm{M}$, grey bar). AITC application (but not heating) resulted in an inward current. b) Current/Voltage relationship from averages of three step protocols done at room temperature (blue trace), during the heat stimulation (red trace), and at the end of AITC application (orange trace; note that the timing of each set of measurements is also labeled as $\mathbf{A}, \mathbf{B}$ and $\mathbf{C}$ on the trace shown in panel A). c) Current density (Max/capacitance) at $32^{\circ} \mathrm{C}$ and in the presence of AITC recorded in mock-transfected, ITRPA1-A transfected, and Smed-TRPA1 transfected cells. Black line = mean; Colored boxes = +- STD; Grey empty boxes = 95\% Confidence Interval. $\mathbf{d}$ ). Dose-response for AITC activation of Smed TRPA1 (AV \pm STD; $n=5$ cells/condition; mustard flower and seed represent an iconic source of AITC). 

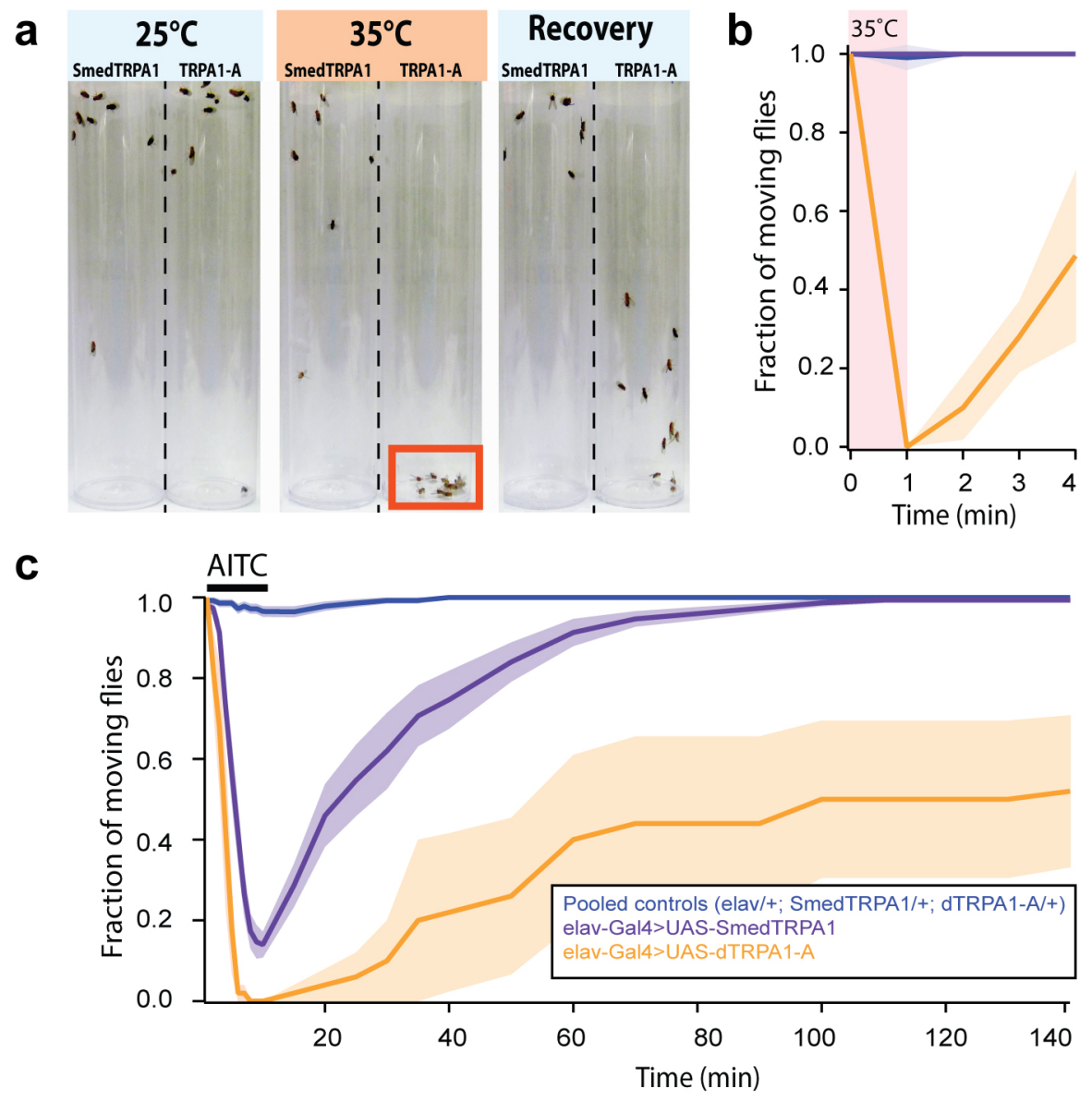

Figure 4: Functional expression of Smed-TRPA1 in vivo in adult Drosophila further demonstrates that the channel is sensitive to AITC but not to heat $\left({ }^{\circ} \mathrm{C}\right)$. a) Adult fruit flies expressing either SmedTRPA1 or -as a control- the intrinsically heat sensitive Drosophila TRPA1-A splice variant, throughout the nervous system (under the control of elav-Gal4) were subjected to a brief step at $35^{\circ} \mathrm{C}(\mathrm{a}$ temperature which does not normally impair fly activity). TRPA1-A expressing flies are readily and reversibly incapacitated by heat (presumably because of simultaneous depolarization of neurons, caused by channel opening) and fall to the bottom of the tube; Smed-TRPA1 flies appear instead unaffected. b) Quantification of the experiment in $\mathbf{a}$. Blue trace = pooled controls (elav/+; UAS-SmedTRPA1/+; UAS-TRPA1-A/+ ; N=4 groups of 10 animals for each, tested separately); purple trace = experimental animals (elav-Gal4>UAS-Smed-TRPA1; $\mathrm{N}=4$ groups of 10 animals); orange trace = positive control (elav-Gal4>UAS-TRPA1-A; $\mathrm{N}=4$ groups of 10 animals; for all traces shaded area \pm SEM). c) Adult flies expressing either Smed-TRPA1 or TRPA1-A were reversibly incapacitated by brief exposure to AITC vapors (see methods for details; Groups and Ns as above; shaded area \pm SEM). 
a

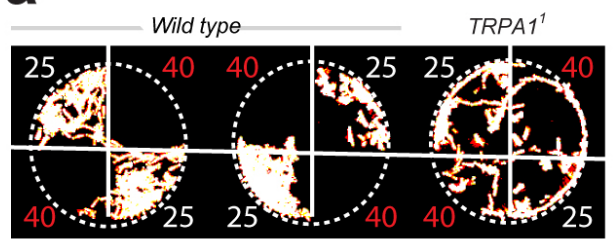

C

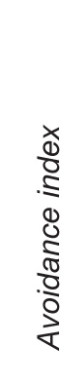

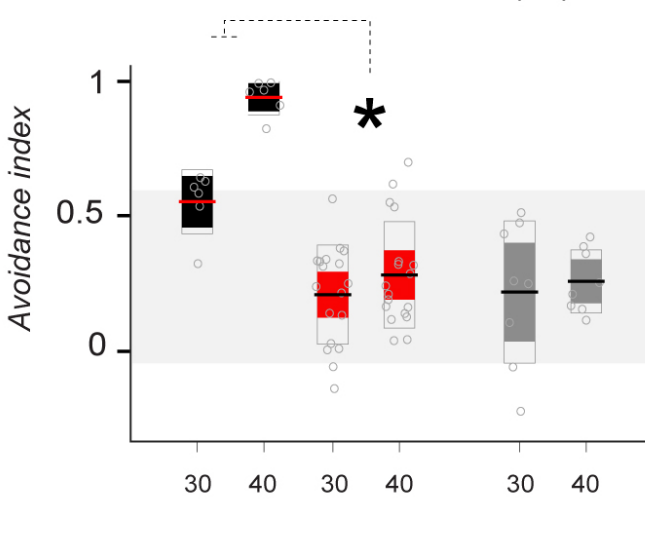

Test Temperature $\left({ }^{\circ} \mathrm{C}\right)$

Figure 5: Across-phylum rescue of Drosophila TRPA1 mutant phenotypes by planarian and human TRPA1. a) In a 2-choice assay, wild type Drosophila flies robustly avoid noxious heat $\left(40^{\circ} \mathrm{C}\right)$. In contrast, TRPA $1^{1}$ mutants explore more readily the $40^{\circ} \mathrm{C}$ quadrants (the panels are maximumprojections of 3 ' movies, illustrating the extent of fly movement, temperature in ${ }^{\circ} \mathrm{C}$ is indicated next to each quadrant). b) Schematic of the rescue experiments. c) Avoidance index of wild-type (black boxes), TRPA $1^{1}$ mutants (red), rescues (yellow, brown, green), and control genotypes (grey). TRPA $1^{1}$ mutants display a significantly lower avoidance index for heat. Pan-neural expression (under the control of elav-Gal4) of mRNA encoding Drosophila TRPA1-C (a splice variant encoding a channel that is not heat-sensitive, yellow), Smed-TRPA1 (brown), or human TRPA1 (green, each under a UAS- promoter) significantly rescues noxious heat avoidance $\left(40^{\circ} \mathrm{C}\right)$. Control genotypes: elav driver/+; TRPA $1^{1}$ and UAS-transgene/+; TRPA ${ }^{1}{ }^{1}$ (see methods for full genotypes). Avoidance index values for each test temperature were compared by unpaired t-tests (wt vs TRPA $1^{1}, \mathrm{P}<0.001$ ) or twoway ANOVAs (for all other genotypes), were asterisks denote a significant interaction between the Gal4 and UAS transgene $\left({ }^{*}=\mathrm{P}<0.01\right)$. Thick line $=$ mean; Colored boxes $=+-$ STD; Grey empty boxes $=95 \%$ Confidence Interval. 
a control

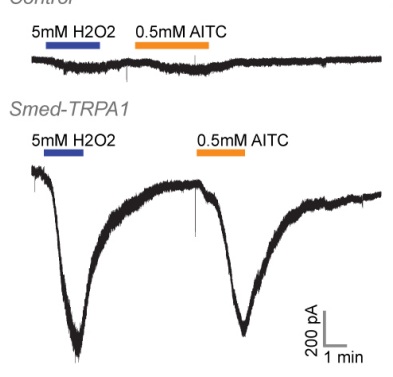

b

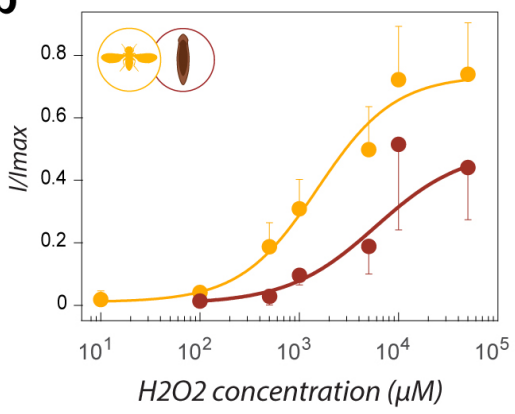

C
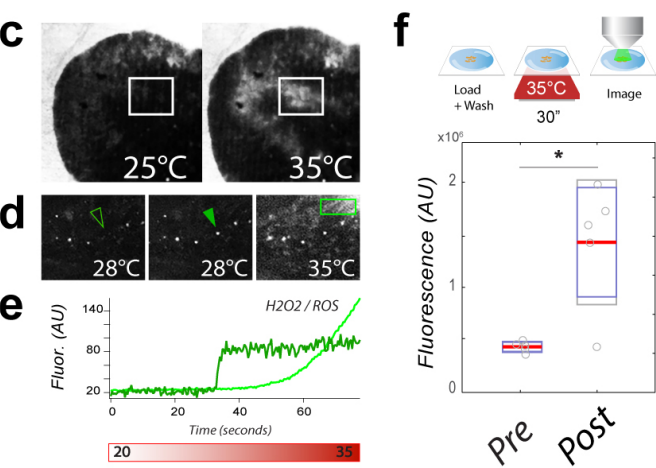

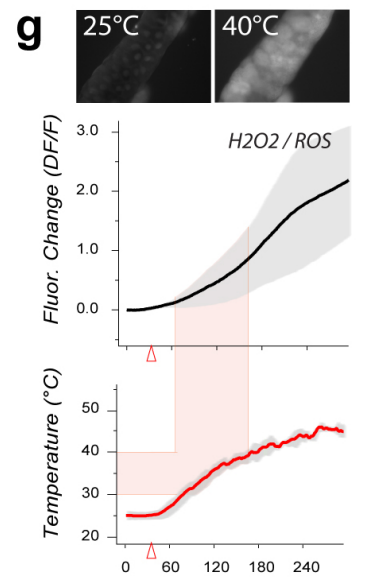

Time (seconds)
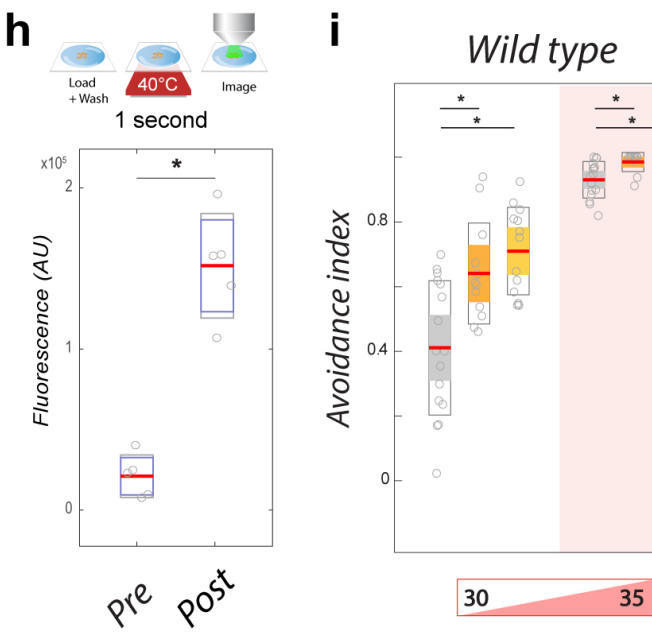

$+/ T R P A 11$

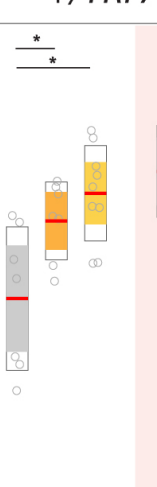

30

Temperature $\left({ }^{\circ} \mathrm{C}\right)$

Figure 6: $\mathrm{H}_{2} \mathrm{O}_{2} /$ ROS as a signal for TRPA1 activation during noxious heat responses. a) Heterologously expressed Smed-TRPA1 is activated by $\mathrm{H}_{2} \mathrm{O}_{2}$. b) Dose-response of $\mathrm{H}_{2} \mathrm{O}_{2}$ activation for Drosophila TRPA1-C (yellow trace and points), and Smed-TRPA1 (brown trace and points, $\mathrm{AV} \pm \mathrm{STD}, \mathrm{n}=5$ cells/condition). c-e) The ROS dye Carboxy- $\mathrm{H}_{2}$ DCFDA demonstrates in vivo ROS production in response to heat in living planarians. $\mathbf{c}$ and $\mathbf{d}$ ) Representative frames of tissues and cells undergoing rapid fluorescent changes in response to heat. e) An ROI around the cell in d (arrow) and the square, light green box are plotted as traces. f) Exposure of planarian worms to $35^{\circ} \mathrm{C}$ for 30" results in a significant increase in fluorescence. g) Carboxy- $\mathrm{H}_{2}$ DCFDA fluorescence in response to heating in Drosophila salivary gland tissue ( $A \bigvee \pm S D)$. $\mathbf{h}$ ) Exposure of salivary glands to $40^{\circ} \mathrm{C}$ for 1 " results in a significant fluorescence increase (in $\mathbf{f}$ and $\mathbf{h},{ }^{*}=\mathrm{P}<0.05 ; \mathrm{n}=5 /$ condition). i) Acute feeding with pro-oxidants sensitizes adult Drosophila to heat. Paraquat (orange bars) or $\mathrm{H}_{2} \mathrm{O}_{2}$ (yellow bars) feeding, results in increased heat voidance in a 2-choice behavioral assay in both wildtype and heterozygous TRPA1/+ controls $\left({ }^{*}=\mathrm{P}<0.05\right)$. In contrast, heat avoidance does not increase in TRPA1 mutants (n.s. =not significant; $\mathbf{n}=11-21$. In $\mathbf{f}, \mathbf{h}$ and $\mathbf{i}$ : Red line = mean; Colored boxes = +STD; Grey empty boxes $=95 \%$ Confidence Interval). 